\title{
Massive Star Astrophysics with the new Magellanic Cloud photometric survey MCSF
}

\author{
Dominik J. Bomans ${ }^{1,2}$, Alexander Becker ${ }^{1}$ and Kerstin Weis ${ }^{1}$ \\ ${ }^{1}$ Astronomical Institute of the Ruhr-University Bochum \\ email: bomans@astro.rub.de, abeck@astro.rub.de, kweis@astro.rub.de \\ ${ }^{2}$ RUB Research Department "Plasmas with complex interactions"
}

\begin{abstract}
Surveys of the resolved stellar content of entire galaxies are the natural tool to study fast evolutionary phases of massive stars. Therefore we launched the Magellanic Clouds Massive Stars and Feedback Survey (MCSF) and periodically imaged for 3 years the entire Small and Large Magellanic Cloud in $\mathrm{u}, \mathrm{B}, \mathrm{V}, \mathrm{R}, \mathrm{I}$ and $\mathrm{H} \alpha$, [OIII], [SII] using the twin telescope RoBoTT at the University Observatory of the Ruhr-University Bochum at Cerro Armazones, Chile. Observations with short exposure times are included to ensure brightest stars not to be saturated, yielding a full coverage in luminosity. With this unique dataset we can study the massive stellar populations up to $M_{B} \sim-10 \mathrm{mag}$ and their feedback. Upon completion a high quality photometric and spatially complete catalog of the Magellanic Clouds will be established which is be comparable (or even beyond) the quality of HST based photometry of nearby galaxies.
\end{abstract}

Keywords. (stars:) supergiants, stars: Wolf-Rayet, (stars:) Hertzsprung-Russell diagram, stars: emission-line, ISM: bubbles, surveys, (galaxies:) Magellanic Clouds, galaxies: stellar content, methods: statistical

\section{Introduction}

Our closest actively star forming neighbor galaxies, the LMC and the SMC are surprime laboratories of stellar evolution and feedback of stars on the interstellar medium. They are nearby enough to be easily resolved into individual stars, are at relatively high galactic latitude (low foreground absorption), have somewhat (LMC) and significantly (SMC) lower metallicity of compared to the Milky Way, and very well determined distances. Still, due to their proximity, they extend on a quite large area on the sky, posing special problems to derive global parameters and compile complete samples in the area of CCDbased optical observing.

Several CCD based survey have already be conducted on the Magellanic Clouds (MCs), but there are some drawbacks to all these data sets. There are saturation problems at the brightest magnitudes (typically above $12 \mathrm{mag}$ ) e.g. in MCPS and OGLE (Zaritsky \& Harris 2004; Zaritsky et al. 2002; Udalski et al. 2008a,b), limitations in terms of filters used, e.g. MCELS (Smith et al. 2000), or the surveys do not cover the whole extend of the Magellanic Clouds (Massey 2002).

This motivated us to undertake a new survey specifically designed to analyse the massive stars and their feedback on the interstellar medium. The survey goal is a coherent data set in both broad band filters (for stellar parameters) and narrow band filters (structure and ionization of the ISM), deep enough to be comparable to HST data set of more distant Local Group galaxies. The idea of MCSF, the "Magellanic Clouds Massive Stars and their Feedback" survey was born.

During this conference several examples of the power of complete datasets were presented, e.g. by P. Massey, E. Levesque, and M. Kourniotis. Another example is the 
recent analysis of Wolf-Rayet stars in the Magellanic Clouds (Hainich et al. 2014). When combining the optical data pf MCSF with similar resolution data in the NIR (2MASS (Skrutskie et al. 2006)), MIR (SAGE (Meixner et al. 2006; Gordon et al. 2011)), UV (GALEX and SWIFT) (Simons et al. 2014; Hagen et al. 2014), or X-ray (XMM-Newton) (Sturm et al. 2013; Bomans 2013), synergy effects allow an even broader set of science projects, as shown below.

\section{Observation and reduction}

The data where taken mostly between 2011 and 2013 with the RoBoTT twin telescopes at the observatory of the Ruhr-University Bochum (Cerro Armazones, Chile). The system is a robotic telescope with predefined queue operation. RoBoTT consists of two Takahashi $15 \mathrm{~cm}$ refractors on a common mount. The Apogee Alta $4096^{2}$ CCDs give a field of view of $2.42^{\circ} \times 2.42^{\circ}$ each, approximately co-spatial on the sky. The resulting pixel scale of the raw images is $2.34^{\prime \prime} /$ pix. We observed two overlapping fields for the SMC covering approximately $4.5^{\circ} \times 2.5^{\circ}$ and 9 fields in the LMC, covering about $8^{\circ} \times 8^{\circ}$ with many slightly dithered exposures spread over the three Magellanic Clouds observing seasons. Images where taken in $\mathrm{u}, \mathrm{B}, \mathrm{V}, \mathrm{R}, \mathrm{I}$, as well as $\mathrm{H} \alpha$, [OIII], and [SII] filters with complete area coverage. Some limited time resolution is possible due to 9 individual visits to the two SMC fields. Due to the large number of fields only few return visits could be scheduled for the LMC. We also took short exposure data in all broad band filters and $\mathrm{H} \alpha$ to ensure non-saturated images of even the brightest Magellanic Cloud member stars.

Basic data reduction was performed with the latest version of the THELI (Erben et al. 2005) pipeline. Making use of the many individual images, we could drizzle the images to a pixel scale of $1^{\prime \prime}$, significantly improving the sampling of our PSF. Pointspread function fitting photometry was performed using a modifed version of DOPHOT (Alonso-Garcia et al. 2012), which allowed a spatially variable PSF. We also performed extensive artificial star experiments to test our completeness. Spatial variation of the crowding in both the LMC and SMC fields is large, but we still reached our goal of better than $90 \%$ completeness in both $\mathrm{B}$ and $\mathrm{R}$ for stars brighter than $~ 19.3$. With these parameters MCSF data of the massive stars and the ionized gas of the Magellanic Clouds are comparable to the HST data of other Local Group galaxies at factor $>10$ larger distances.

\section{First science results}

Currently we are in the optimisation phase of the data reduction, therefore only a few glimpses of what we plan to do and already started to do with MCSF are presented here.

\subsection{The Magellanic Clouds as external galaxies: Color magnitude/Hess diagrams}

In Fig.1 we plot the B-R,B color magnitude diagrams of the LMC (left) and SMC (right). By color coding the density of source at each position we extend the CMDs to Hess diagrams (Hess 1924). These diagrams allow not only to study the location of structures in the CMD, but also the number density of stars in the corresponding evolutionary phase. This enables us studies of the spatial distribution of stars in the same evolutionary phase and (using stellar evolution grids) their spatial change with evolution time. The MCSF CMDs show a split of the apparent main sequence and the blue supergiants. The tip of the red supergiants is well defined, but some stars are apparently located above the predictions of stellar evolution (Levesque et al. 2006) and the Humphreys-Davidson limit (Humphreys \& Davidson 1979). The tip of the RGB and the extension towards very red stars (AGB, carbon stars) are also clearly visible. 

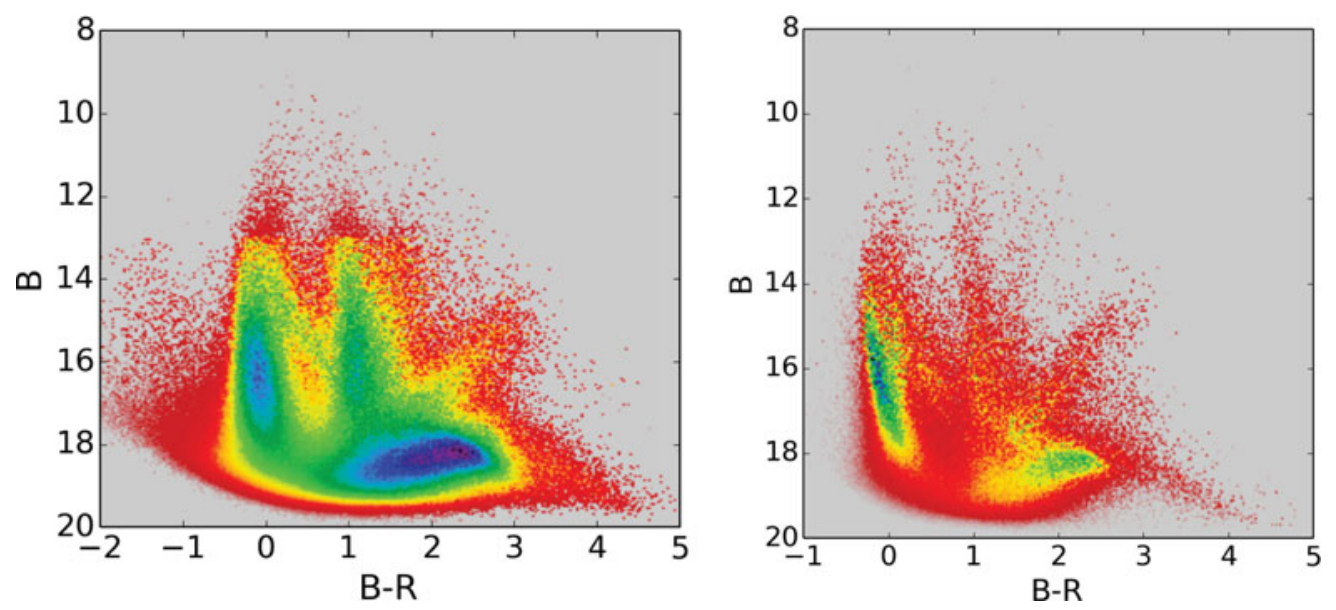

Figure 1. MCSF based B-R,B color magnitude diagrams of the whole LMC and SMC, with added color / grayscale coding for the number density of the stars at the position in the diagram (Hess 1924).

The region between the main sequence and red supergiant branches is dominated by the galactic foreground. Correction of this unwanted feature is the most critical point to be improved, yet. The CMDs plotted in Fig. 1 are not strongly cleaned for photometric error or shape parameters in order to give a full impression of the data. Clearly some areas (e.g. the very blue objects in the LMC CMD) are artifacts.

\subsection{Be stars}

With not only broad band filter photometry in hand, but also deep $\mathrm{H} \alpha$ and [OIII], we can analyse the emission line stars content of both Magellanic Clouds. We use our photometric catalog in $\mathrm{B}$ and $\mathrm{R}$ to merge it it with the source catalog in $\mathrm{H} \alpha$. We then rejected all objects which are bright in [OIII] as probable compact HII regions. Afterwards we use the color - magnitude diagram to define the locus main sequence stars and supergiants.

The resulting histogram of the ratio of emission line objects normalized to total objects as function of brightness is plotted in the left panel of in Fig.2. As observed several times before (e.g. Iqbal \& Keller 2013), the main sequence Be/B star ratio is higher in the SMC than in the LMC. The difference in our data seems to be larger for the Oe stars than the Be stars and the ratio is about constant from B0 to the later B stars, or even slightly rising going to lower masses. The absolute number of the the $\mathrm{Be} /(\mathrm{Be}+\mathrm{B})$ ratio we derive from our data is smaller than the ratio derived by Martayan et al. (2010), but consistent with the value derived by Iqbal \& Keller (2013). The reason for this difference may be that slitless spectroscopy used in Martayan et al. (2010) is more efficient to detect faint Balmer emission than the narrow band filter method employed by us. Another explanation of the difference may be related to the definition of the main sequence.

Beside checking our selection criteria used, and performing a star by star comparison with the samples of Sabogal et al. (2005); Martayan et al. (2010), we will also look into differences between clusters and field (Keller et al. 1999), color effects, and variability (Beaulieu et al. 2001; Keller et al. 2002) of our sample in comparison to earlier ones. With the full coverage and good photometry at hand we will also look at the Oe stars and supergiant emission line stars and investigate the apparent downturn of the ratio in the LMC in the $\mathrm{O}$ emission line star regime. This downturn appears to be much weaker in the SMC (see Fig.2a). 

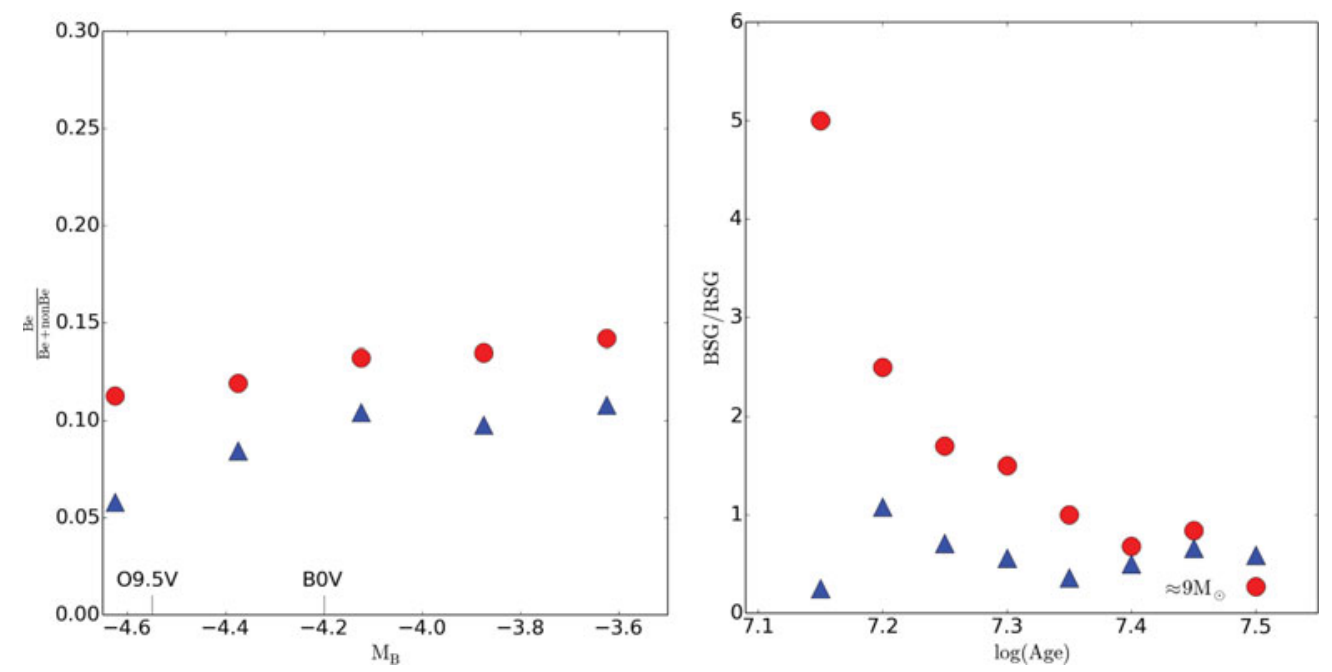

Figure 2. (a) Ratio $\mathrm{Oe} /(\mathrm{Oe}+\mathrm{O})$ and $\mathrm{Be} /(\mathrm{Be}+\mathrm{B})$ star ratio for LMC and SMC based on MCSF $\mathrm{H} \alpha$ and broad band photometry. LMC data are plotted as triangles, SMC data are plotted as circles. (b) Blue to red supergiant ratio of of the LMC and SMC. Markers are the same as the left panel.

\subsection{Blue to red supergiant ratio}

The stars going in their evolution trough both red and blue supergiant phase are exactly the stars which are progenitors to the majority of the type II supernovae. Understanding their evolution is critical, since they provide the largest part of the kinetic energy feedback into the interstellar medium and a significant part of the heavy metals. This ratio depends on a large number of aspects of stellar structure and evolution (e.g. Maeder \& Meynet 2001). MCSF is a well suided database to explore the the physics of this ratio. For a first attempt, we counted stars along lines of same age using Geneva isochrones (Lejeune \& Schaerer 2001; Georgy et al. 2013) and alternatively used the cuts along the same luminosity to derive the ratios. The results of the first method are plotted in Fig. $2 \mathrm{~b}$. The SMC shows higher BSG/RSG ratio than the LMC in all mass bins, exept the very lowest. Interstingly, the difference between the SMC and LMC ratio appears to decrease with decreasing mass. While the models with rotation are a much better fit to the ratios than models without rotation, the agreement between observation and models is still not satisfactory. Either the models miss some relevant physics, or our selection criteria do not yet catch stars at well defined ages, or both.

\subsection{Wolf-Rayet and other hot evolved stars}

Supplementing the MCSF multiband photometry with UV (GALEX, SWIFT) und IR (2MASS, Spitzer) data creates a large set of very wide stellar spectral energy distribution (SED). This data set we crossmatched with the "Catalogue of Stellar Spectral Classifications" (Skiff, B. A. 2013) generating a sample of Magellanic Cloud stars with known spectral classification. This sample was used to train a machine learning algorithm that is able to estimate an "semi-spectral" classification of a star based on its SED. The algorithm is able to reproduce $98 \%$ of the training sample. We applied it to $\sim 36000$ stars in the SMC and $\sim 45000$ stars in the LMC to assess their "spectral" type. First tests of this method showed a generally good agreement within a few spectral subtypes. We used the derived classifications to compile a list of 150 new Wolf-Rayet stars and very hot supergiant candidates in the LMC and SMC. A comparison with the Wolf-Rayet stars found in 
a new search program by Massey et al. (2014) showed that the astro-informatics method is able to find new Wolf-Rayet stars: in the very small overlap between our sample and the Massey et al. (2014) sample, we correctly predicted one (LMC143-1) of two new spectroscopically confirmed Wolf-Rayet stars. Both our MCSF plus astro-informatics based result and the search project by Massey et al. (2014) point at the same direction: The inventory of the Magellanic Clouds Wolf-Rayet stars is far from complete, yet.

\section{The archive}

We set up a dedicated web site for MCSF to serve as first stop for status, results, and the actual data: http://www.astro.rub.de/Astronomie/MCSF. At the time of writing (summer 2014) we are in the middle of quality tests for our photometry. We already started to upload technical details, some plots, and pretty pictures to the archive page. Starting from fall 2014, we plan to upload the first photometric catalogs and ancilliary data. Full catalogs and finally the images will follow, as we write science papers. In case you need some data fast, please do not hesitate to contact us.

\section{References}

Alonso-Garcia, J., Mateo, M., Sen, B., et al. 2012, AJ 143, 70

Beaulieu, J.-P., de Wit, W. J., Lamers, H. J. G. L. M., et al. 2001, A\&\&A 380, 168

Bomans, D. J. 2013, in G. Pugliese, A. de Koter, \& M. Wijburg (eds.), 370 Years of Astronomy in Utrecht, Vol. 470 of ASP Conference Series, p. 245

Erben, T., Schirmer, M., Dietrich, J. P., et al. 2005, Astron. Nachr. 326, 432

Georgy, C., Ekström, S., Eggenberger, P., et al. 2013, A\&A 558, A103

Gordon, K. D., Meixner, M., Meade, M. R., et al. 2011, AJ 142, 102

Hagen, L., Siegel, M., Gronwall, C., Hoversten, E. A., \& Immler, S. 2014, in American Astronomical Society Meeting Abstracts \#223, Vol. 223, p. 442.30

Hainich, R., Rühling, U., Todt, H., et al. 2014, A\& A 565, A27

Hess, R. 1924, in Probleme der Astronomie. Festschrift fur Hugo v. Seeliger, p. 265, Springer, Berlin

Humphreys, R. M. \& Davidson, K. 1979, ApJ 232, 409

Iqbal, S. \& Keller, S. C. 2013, MNRAS 435, 3103

Keller, S. C., Bessell, M. S., Cook, K. H., Geha, M., \& Syphers, D. 2002, AJ 124, 2039

Keller, S. C., Wood, P. R., \& Bessell, M. S. 1999, A\&AS 134, 489

Lejeune, T. \& Schaerer, D. 2001, A\&A 366, 538

Levesque, E. M., Massey, P., Olsen, K. A. G., et al. 2006, ApJ 645, 1102

Maeder, A. \& Meynet, G. 2001, A\& $A$ 373, 555

Martayan, C., Baade, D., \& Fabregat, J. 2010, A\&A 509, A11

Massey, P. 2002, ApJS 141, 81

Massey, P., Neugent, K. F., Morrell, N., \& Hillier, D. J. 2014, ApJ 788, 83

Meixner, M., Gordon, K. D., Indebetouw, R., et al. 2006, AJ 132, 2268

Sabogal, B. E., Mennickent, R. E., Pietrzyński, G., \& Gieren, W. 2005, MNRAS 361, 1055

Simons, R., Thilker, D., Bianchi, L., \& Wyder, T. 2014, Advances in Space Research 53, 939

Skiff, B. A. 2013, VizieR Online Data Catalog 1, 2023

Skrutskie, M. F., Cutri, R. M., Stiening, R., et al. 2006, AJ 131, 1163

Smith, C., Leiton, R., \& Pizarro, S. 2000, in D. Alloin, K. Olsen, \& G. Galaz (eds.), Stars, Gas and Dust in Galaxies: Exploring the Links, Vol. 221 of ASP Conference Series, p. 83

Sturm, R., Haberl, F., Pietsch, W., et al. 2013, A\&A 558, A3

Udalski, A., Soszynski, I., Szymanski, M. K., et al. 2008a, AcA 58, 89

Udalski, A., Soszyński, I., Szymański, M. K., et al. 2008b, AcA 58, 329

Zaritsky, D. \& Harris, J. 2004, ApJ 604, 167

Zaritsky, D., Harris, J., Thompson, I. B., Grebel, E. K., \& Massey, P. 2002, AJ 123, 855 


\section{Discussion}

MAssey: Absolutely beautiful work - I'm glad to see someone doing this right. One question: I'm surprised by the number of very luminous red supergiant candidates you have. In Neugent et al. (2012) we don't see any of these. I'm wondering of you check the proper motions from the UCAC4 survey? This might help weed out the foreground stars.

Bomans: Up to now, we tried to correct for the foregound by using statistical subtraction from Magellanic Cloud star free (or nearly so) parts of fields. Both this and using predictions from galactic models did not work fully satisfactory. So, I guess a lot of the RSG candidate may well be foreground stars. Thanks for the suggestion, we will try with the proper motions data to clear this CMD area.

MAEDER: The comparison of the numbers of blue to red supergiants are very important. As said long ago by Kippenhahn: this is a magnifying glass of all the mistakes we have made in the models. As a side remark, I point out that AGB at the top luminosity may influence the distribution of luminosities.

Bomans: We are working to improve our selection of especially the blue supergiants to make the blue to red supergiant ratios even cleaner from interlopers. The effect of the brightest AGB star contributon to the RSG numbers may explain the small bump in the BSG/RSG ratio we see near the luminosity of the faintest RSGs. Thanks for the comment.

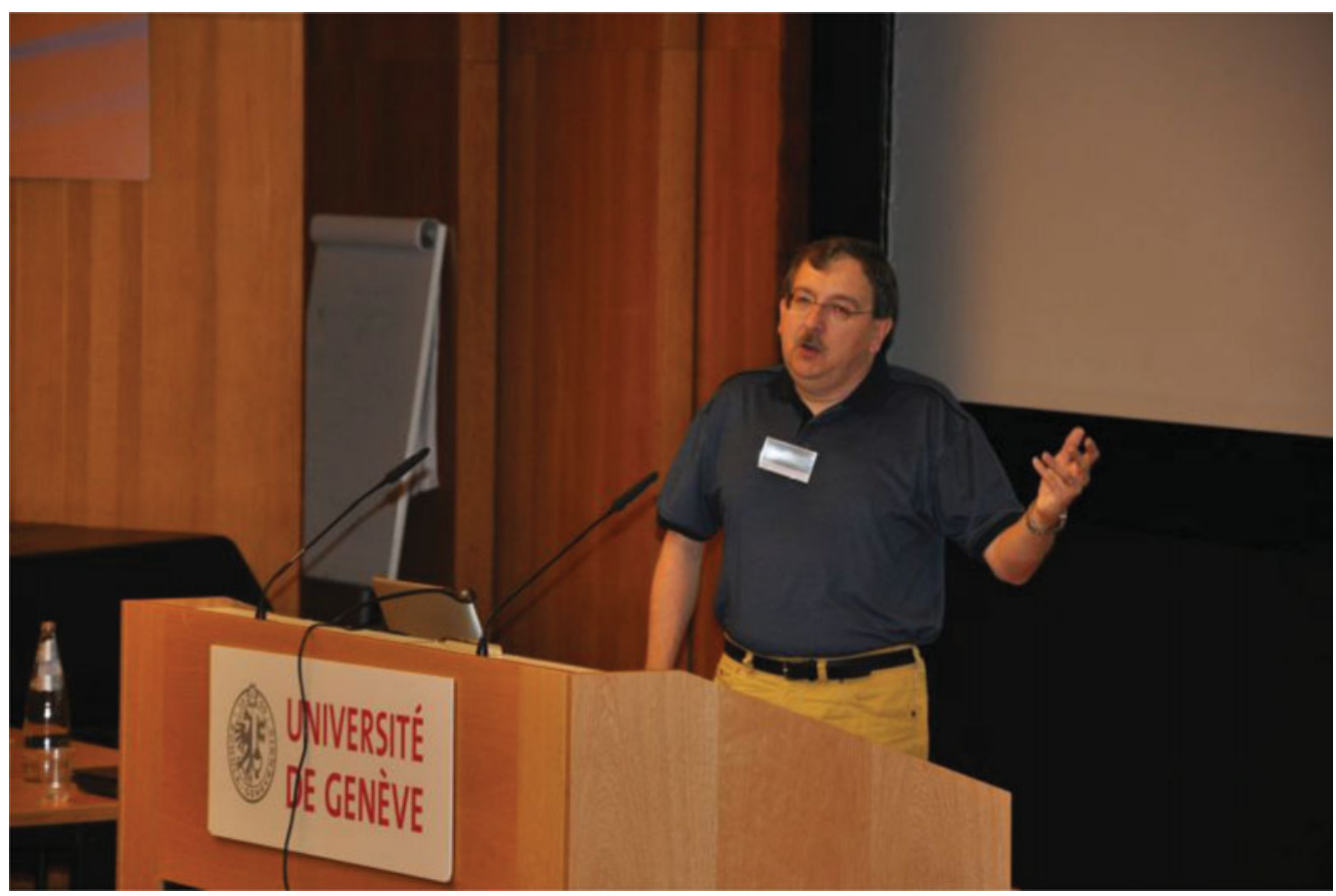

Dominik Bomans 